\title{
Salivary IgA against sporozoite-specific embryogenesis-related protein (TgERP) in the study of horizontally transmitted toxoplasmosis via $T$. gondii oocysts in endemic settings
}

\author{
B. M. MANGIAVACCHI ${ }^{1} \uparrow$, F. P. VIEIRA ${ }^{1} \dagger$, L. M. G. BAHIA-OLIVEIRA ${ }^{2 *}$ \\ AND D. HILL ${ }^{3}$ \\ ${ }^{1}$ Laboratório de Biologia do Reconhecer, Centro de Biociências e Biotecnologia, Universidade Estadual do Norte \\ Fluminense Darcy Ribeiro, Campos dos Goytacazes, Rio de Janeiro, Brazil \\ ${ }^{2}$ Laboratório de Imunoparasitologia, Universidade Federal do Rio de Janeiro, Macaé, Rio de Janeiro, Brazil \\ ${ }^{3}$ Animal Parasitic Diseases Laboratory, Beltsville Agricultural Research Center, United States Department of \\ Agriculture, Beltsville, MD, USA
}

Received 14 December 2015; Final revision 1 April 2016; Accepted 19 April 2016; first published online 12 May 2016

\section{SUMMARY}

The aim of this study was to contribute to the better understanding of the relative epidemiological importance of different modes of infection with respect to horizontal transmission of Toxoplasma gondii in endemic settings. We investigated the prevalence of salivary IgA against a sporozoite-specific embryogenesis-related protein (TgERP) in a highly endemic area for toxoplasmosis in Brazil in order to pinpoint parasite transmission via oocysts. Prevalence calculated by salivary IgA specific to TgERP was compared to the prevalence calculated by serum IgG against both TgERP and tachyzoites (in conventional serological tests). Prevalence calculated by different serological and salivary parameters varied in the studied age groups. However, for the 15-21 years age group, values for $T$. gondii prevalence estimated by conventional serological tests and by anti-TgERP salivary IgA were similar; i.e. $68 \cdot 7 \%$ and $66 \cdot 6 \%$ or $66.7 \%$, respectively, using two different cut-off parameters for salivary IgA anti-TgERP.

Furthermore, salivary IgA anti-TgERP for this age group presented the highest specificity $(93.33 \%)$, sensitivity (93.94\%), and likelihood (14.09) compared to all the other age groups. These data demonstrate the importance of age for salivary $\operatorname{IgA}$ investigation against TgERP to estimate the mode of $T$. gondii transmission in endemic settings.

Key words: Diagnosis, oocysts, salivary IgA, Toxoplasma gondii, waterborne infections.

\section{INTRODUCTION}

Toxoplasmosis infection caused by the protozoan Toxoplasma gondii is widely distributed in humans in many parts of the world [1]. In Brazil, its prevalence ranks among the highest in the world [2]. A broad

\footnotetext{
* Author for correspondence: Dra L. M. G. Bahia-Oliveira, Laboratório de Imunoparasitologia, Universidade Federal do Rio de Janeiro, Macaé, Rio de Janeiro, Brazil. (Email: lilian@uenf.br)

$\dagger$ These authors contributed equally to this work.
}

range of potential routes of $T$. gondii transmission has been developed by the parasite in the course of its evolution [3]. Congenital toxoplasmosis in humans results from vertical transmission of the parasite during pregnancy and constitutes a very dangerous mode of infection to the fetus, causing stillbirth or a variety of disease syndromes, from chronic infection without apparent clinical symptoms, to blindness and mental retardation in children [4, 5]. Besides congenital infection, humans become infected through ingestion of tissue cysts in undercooked or uncooked 
meat, or by ingesting food or water contaminated with sporulated oocysts (containing the infective sporozoite forms) from infected feline faeces [6-12]. The relative epidemiological importance of different modes of infection with respect to horizontal transmission of $T$. gondii, namely by ingestion of tissue cysts in undercooked or uncooked meat, or by ingesting food or water contaminated with sporulated oocysts from cat faeces [13-16], remains unknown. The ability to discriminate between the two modes of infection depends, among other factors, on serological tests capable of discriminating both modes of infection. In epidemiological terms, using conventional serology, which recognizes antigens from tachyzoites, it is not possible to determine the predominant route (oocysts or tissue cysts) of transmission [17]. Serological diagnosis pinpointing $T$. gondii cysts or oocysts as the mode of transmission by means of an ELISA using a recombinant protein, i.e. the sporozoite-specific embryogenesis-related protein (TgERP) [18], and more recently the CCp5A recombinant protein [19], has allowed differentiation between the two modes of infection.

IgA antibodies together with $\operatorname{IgM}$ and $\operatorname{IgG}$ against antigens derived from tachyzoite forms of $T$. gondii are frequently detected in sera of patients in the early and chronic phase of toxoplasmosis [20]. The presence of $\operatorname{IgG}$ and $\operatorname{IgA}$ anti- $T$. gondii in saliva and other body fluids has also been reported [21-25]. However, the specific role of IgA against $T$. gondii in the human oral cavity remains elusive. The isolation and/or detection of viable $T$. gondii from human saliva has also been reported [21, 26, 27], thus the presence of $\operatorname{IgA}$ against sporulated $T$. gondii oocyst antigens in the saliva of patients exposed to the risk of $T$. gondii infection is plausible and an open question for investigation. In the present study T. gondii prevalence using conventional and TgERP ELISAs was investigated in the sera and saliva of patients exposed to the risk of $T$. gondii infection via oocyst infection [20]. It was found that salivary IgA against TgERP is an important antibody to be considered in the study of horizontal $T$. gondii infection transmitted by oocysts.

\section{METHODS}

\section{Study population}

The subjects enrolled in the present study were from a previously studied endemic area for toxoplasmosis
[28]. In terms of socioeconomic status, they correspond to the lower and lower/middle-class income groups [28]. For sample size calculation (confidence level of $95 \%$ and margin of error of 5\%) it was considered the seroprevalence reference of $73 \cdot 1 \%$, which is the expected frequency for lower and lower/middleclass income groups as previously described [28, 29]; the required sample size was 302 subjects. A total of 476 individuals associated (as students, school staff, students' parents) with 10 public schools from Campos dos Goytacazes, located in the north of Rio de Janeiro state (Table 1), were tested in the current study. Schools were representative of the city in terms of geographical locations (urban, suburban, or rural areas) [28]. Individuals were invited to participate in the study; consent was sought and granted (self or guardian) after individuals were informed about the project. Five millilitres of blood was collected from each individual for serological assay. Saliva was collected under stimulation by rubbing the gums with a Salivette sampler sponge (Sarstedt AG \& Co., Germany) for $\sim 1$ min. After centrifugation, saliva samples were separated in aliquots prior to storage. Each clinical sample (sera and saliva) was labelled to preserve individual identification codes and kept at $-20{ }^{\circ} \mathrm{C}$ until use. In a group of 96 individuals aged $>28$ years, members of the adult school staff, only $5 \mathrm{ml}$ of blood sample was collected (no saliva sample was collected) (Table 1). Standard serological tests [Vidas Toxo IgG and IgM kits (bioMérieux, France) and Architect Toxo IgG and IgM kits (Abbott Diagnostics Division, USA)] that are able to detect previous $T$. gondii exposure without discriminating the route of infection were performed to evaluate acute and chronic $T$. gondii human infection. Subjects included in control groups were those that tested negative for conventional ELISA against $T$. gondii (IgG and or IgM) and varied according to age group: $0-7$ years ( $n=104$ subjects), $8-14$ years $(n=99), 15-21$ years $(n=15)$, and $22-28$ years $(n=15)$.

\section{ELISA against TgERP}

The sera and saliva of 380 subjects were analysed for reactivity against TgERP [18]. Briefly, testing for reactivity to TgERP was performed by ELISA; TgERP (uncleaved with factor $\mathrm{Xa}$ ) was diluted to a concentration of $2 \mu \mathrm{g} / \mathrm{ml}$ in $0 \cdot 1 \mathrm{M}$ carbonate buffer ( $\mathrm{pH}$ 9.6). In sequence, plates were washed three times with wash buffer [50 mM Tris ( $\mathrm{pH} 7 \cdot 4$ ), 


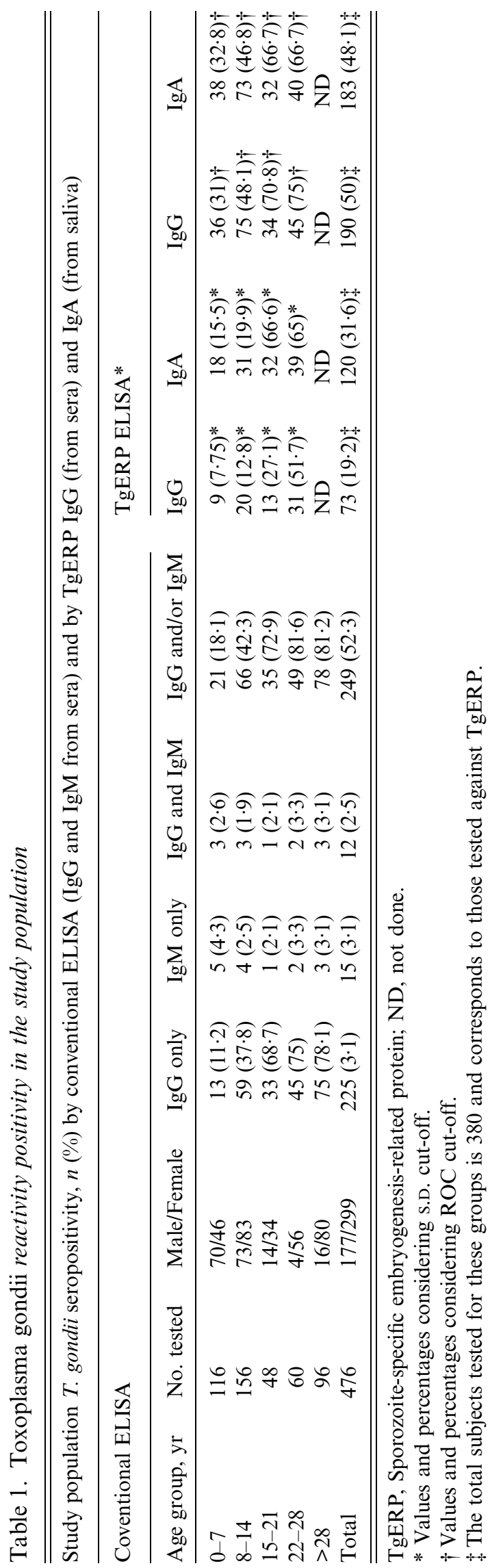

$150 \mathrm{~mm} \mathrm{NaCl}, 5 \%$ skim milk, $1 \cdot 0 \%$ Triton X-100]. The plates were then incubated with $100 \mu \mathrm{l} /$ well of prediluted sera or saliva (1:100 and 1:10, respectively) in wash buffer for $30 \mathrm{~min}$ at room temperature. After another cycle of three washes, $100 \mu$ of rabbit immunoglobulin anti-human IgG (for sera samples) or anti-human IgA (for saliva samples) conjugated to peroxidase (Kirkegaard \& Perry Laboratories Inc., USA) pre-diluted 1:1000 in wash buffer was added to sera and saliva tests, respectively. The plates were incubated for $30 \mathrm{~min}$ at room temperature and then washed again three times with wash solution and incubated with $100 \mu \mathrm{l} /$ well of developing solution. The reading was performed in an ELISA reader (VMax ELISA reader, Molecular Devices Inc., USA) at $405 \mathrm{~nm}$. Reference positive and negative controls were included on each plate for cut-off calculations.

\section{Cut-off calculations for ELISA against TgERP}

The cut-off calculation based on standard deviation (s.D.) was established from a positive cut-off as the mean value of optical density (OD) of seronegative samples in conventional ELISA, plus three times the S.D. of seronegative samples as described previously [18]. Cut-off calculation was based on the receiveroperating characteristic (ROC) curve: the ROC curve data provided the accuracy of each test with area under the curve (AUC) measurements.

sensitivity $=\frac{\text { true positives }}{\text { true positives }+ \text { false negatives }} \times 100$,

specificity $=\frac{\text { true negatives }}{\text { true negatives }+ \text { false positives }} \times 100$,

likelihood ratio $=[$ sensitivity $/(1-$ specificity $)]$

were also calculated for each immunoglobulin reactivity pattern.

\section{Statistical analysis}

Statistical analyses were performed by GraphPad Prism v. 6.0 software (Graphpad Software, USA) for all statistical analyses (ROC curve and Spearman analyses).

\section{Ethical standards}

The study protocol complied with the Declaration of Helsinki and was approved by the local ethics 
committee (protocol nos. 164525/027/07 and 716 $147 /$ 2014).

\section{RESULTS}

\section{Study population characteristics and prevalence of} anti- $T$. gondii IgM and IgG by conventional serology

The mean age of patients was 17.89 years (s.D. = $14 \cdot 79,95 \%$ confidence interval $16 \cdot 56-19 \cdot 23$, range 0-75 years); $177(37 \cdot 2 \%)$ were males and 299 (62.8\%) females (Table 1). The overall seroprevalence of $T$. gondii to IgG and/or IgM antibodies tested by conventional ELISA was $52 \cdot 3 \%(249 / 476)$. Of this, IgG antibodies were detected in $49.8 \%$ (237/476) while IgM antibodies were present in 5.7\% (27/476). Twelve $(2 \cdot 5 \%)$ participants tested positive for both IgG and IgM antibodies. Fifteen (3.1\%) participants tested positive for only IgM antibodies while 47.3\% (225/476) participants tested positive for only IgG antibodies. The remaining $47 \cdot 7 \%$ (227/476) of participants tested negative for all antibodies. A progressively increased statistically significant $(P<0.0001)$ anti- $T$. gondii seropositivity ( $\mathrm{IgG})$ was observed as age increased (Table 1). For IgM, however, the prevalence was not associated with age $(P=0.9284)$.

\section{ROC curves for anti-TgERP IgG and IgA reactivity}

The ability of anti-TgERP IgG in sera samples and IgA in saliva samples to discriminate seropositive populations (by conventional ELISA) was analysed by ROC curves (Fig. 1a,b). The 1:100 serum dilution was selected to measure IgG, whereas 1:10 saliva dilution was the best for discriminatory analysis of IgA. IgA reactivity was the most promising biomarker for discrimination between positive and negative individuals and those who were negative for toxoplasmosis by conventional ELISA. The IgA and $\operatorname{IgG}$ analysis by ROC curves presented good performances in general for all age groups. However, for the $0-7$ years age group the cut-off values established by the ROC curve from saliva $\operatorname{IgA}$ is below the level of the ELISA's technical sensitivity capacity (OD $=0 \cdot 074)$. A good performance was observed for $\mathrm{IgG}$ sera samples for the 15-21 years age group $(\mathrm{Se}=90 \cdot 91 \%, \mathrm{Sp}=$ $73 \cdot 33 \%, \mathrm{AUC}=0.8596$ and $\mathrm{LR}+=3 \cdot 409)$ (Fig. 1a, b). However, the best performance for the 15-21 years age group was found for IgA saliva samples $(\mathrm{Se}=$ $93.94 \%, \quad \mathrm{Sp}=93.33 \%, \quad \mathrm{AUC}=0.9677$ and $\mathrm{LR}+=$ 14.09) compared to all other age groups.

\section{Prevalence of anti-TgERP IgG and IgA according to cut-off determination and Spearman's correlation between sera IgG and saliva IgA anti-TgERP}

The seroprevalence by conventional ELISA IgG and TgERP ELISA (IgG from sera and IgA from saliva) are shown in Table 1 and Figure 2(a,b). The prevalence of IgG measured by antibodies against TgERP reached $75 \%$ in individuals aged $22-28$ years; the prevalence measured by conventional ELISA in the same age group is $81.6 \%$ (Table 1 ). In Figure $2(a, b)$, the prevalence curves are shown as a function of the age group population. The seroprevalence indicated in the TgERP ELISA was calculated considering two cut-off criteria: (1) the threefold standard deviation (S.D. cut-off) values of samples negative in conventional ELISA as published previously $[18,30]$ and (2) by ROC curve (ROC cut-off). The prevalence curves for conventional ELISA IgG and TgERP $\mathrm{IgG}$ and $\operatorname{IgA}$ presented a generally similar shape even when considering S.D. cut-off or ROC cut-off for TgERP ELISA (Fig. 2a, b) with diverging points in all age groups with exception of the 15-21 years age group. For this age group the prevalence values were very similar: for IgG anti- $T$. gondii conventional ELISA (68.7\%), and for IgA anti-TgERP ELISA $(66.6 \%$ and $66 \cdot 7 \%)$ when considering S.D. cut-off and ROC curve cut-off, respectively (Table 1 ). This age group is included in the section of the age prevalence curve where the slope is highest for both conventional ELISA (IgG) and TgERP ELISA (IgG and IgA) (Fig. $2 a, b$, respectively). For all other age groups the $T$. gondii prevalence curves estimated by TgERP ELISA using S.D. cut-off criteria for $\operatorname{IgG}$ and $\operatorname{IgA}$ measurements were lower than the prevalence estimated by ROC cut-off criteria (Table 1). The very low prevalence of IgM estimated by conventional ELISA detected in the present study is shown by a dotted line in Figure 2( $a, b)$. Spearman's correlation test between ELISA OD values of anti-TgERP IgG from sera and anti-TgERP IgA from saliva was used to estimate strength of the link between systemic IgG and salivary IgA, both against TgERP. The best significant correlation parameters were observed for the 15-21 years age group $(r=0.56$ and $P<$ $0 \cdot 0001)$. Considering prevalence calculated by $\operatorname{IgG}$ anti-TgERP $(70 \cdot 8 \%)$ using ROC cut-off values (shown in Table 1) in this age group, it is probable that the cut-off values for TgERP based on ROC curves may be better than the S.D. cut-off for ages $>7$ years. 
(a)

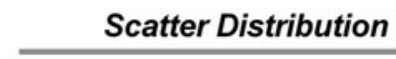

0-7 years (116)

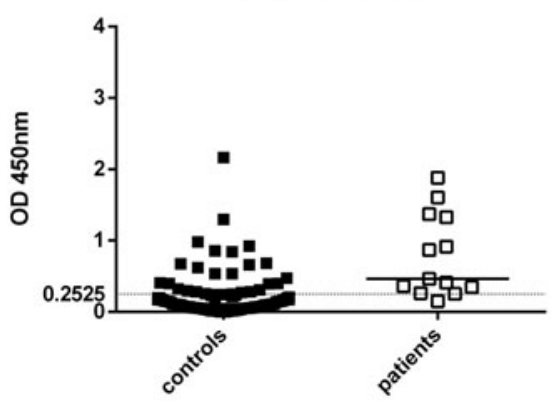

8-14 years (156)

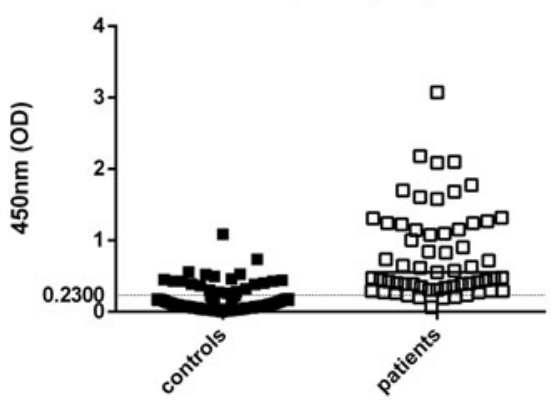

15-21 years $(48)$

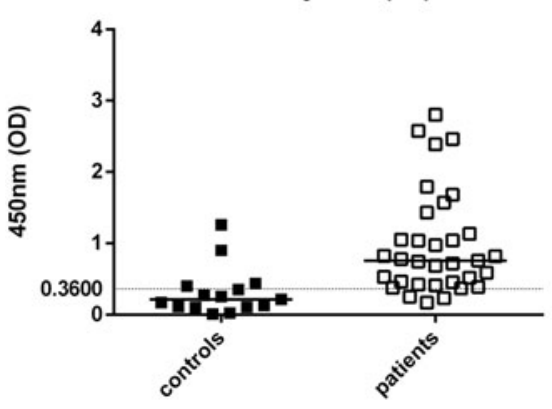

22-28 years $(60)$

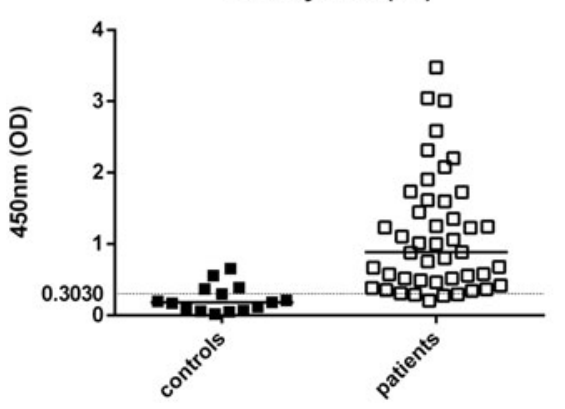

ROC Curve
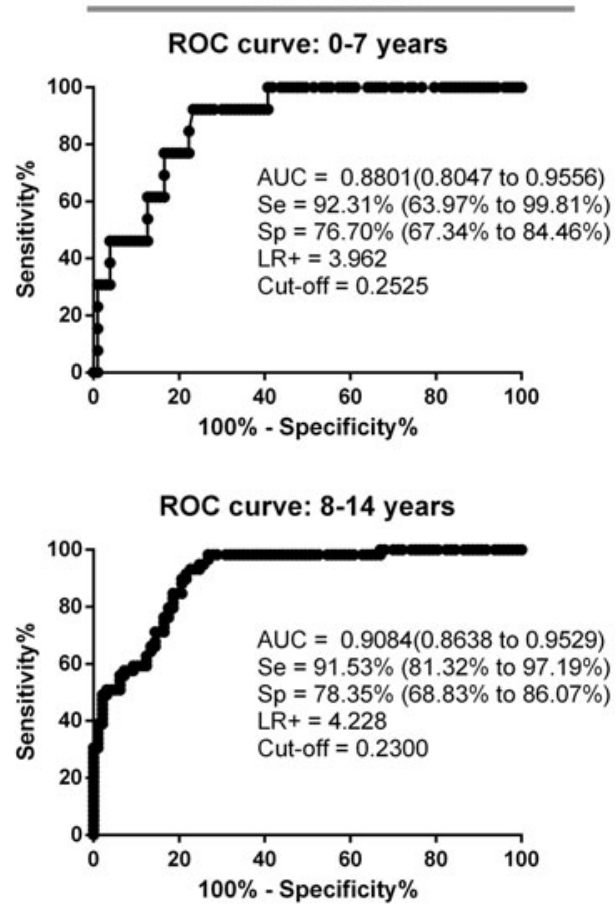

ROC curve: $15-21$ years

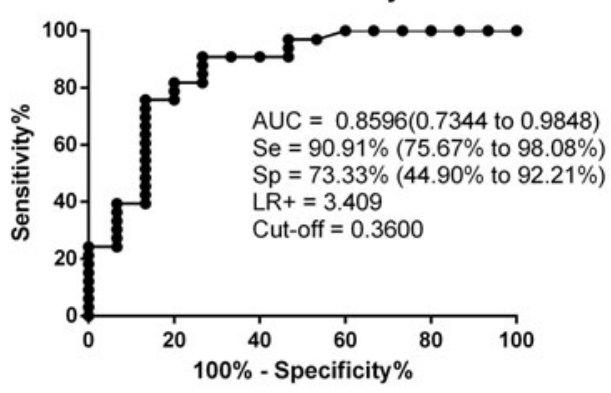

ROC curve: $22-28$ years

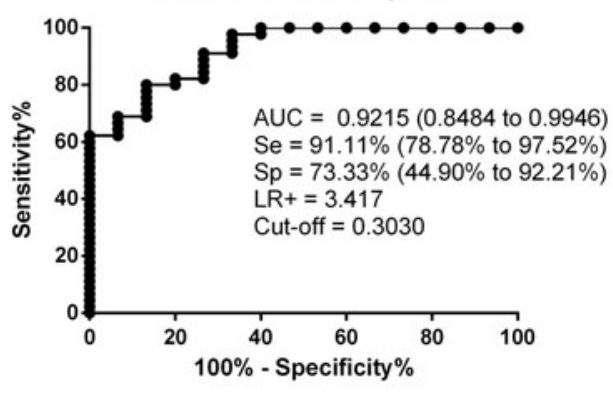

Fig. 1. Anti-Toxoplasma gondii immunoglobulins detected by conventional ELISA (IgG) and sporozoite-specific embryogenesis-related protein (TgERP) ELISA (IgG and IgA) to discriminate controls ( $\square$ ) from patients ( $\square$ ) in the different age groups for the individuals enrolled in the study. Data are expressed as scattering distribution of optical density (OD) for all immunoglobulins. ROC curve analysis was used to establish the best cut-off edge to segregate immunoglobulin reactivity in the groups. The cut-off edges and the performance indices (Se, Sp, AUC and LR+) are provided. (a) IgG reactivity assessed in sera samples for TgERP ELISA. (b) IgA reactivity assessed in saliva samples for TgERP ELISA. The number of individuals in each group is denoted within parentheses. The subjects included in control groups were those that tested negative for conventional ELISA against $T$. gondii (IgG and/or IgM) and varied according to age group: $0-7$ years $(n=104$ subjects), 8-14 years $(n=99), 15-21$ years $(n=15)$, and 22-28 years $(n=15)$. 
(b)

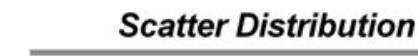

0-7 years $(116)$

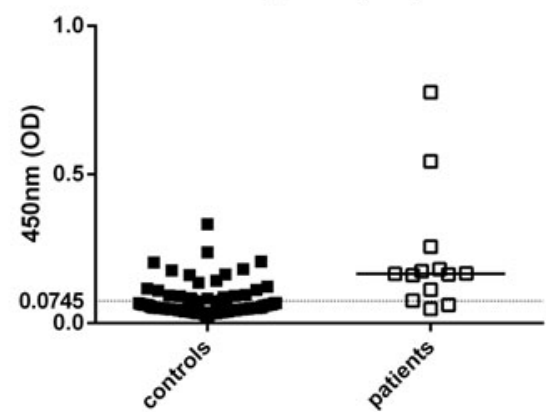

8-14 years $(156)$

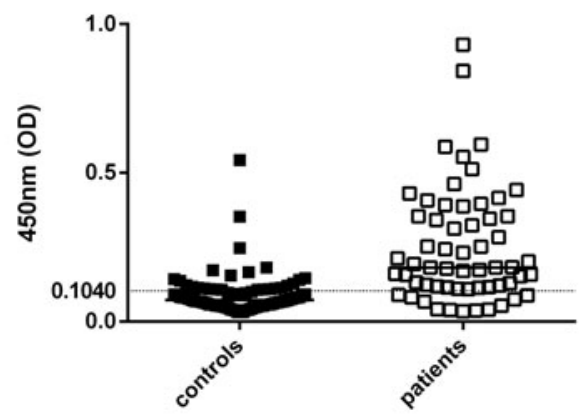

$15-21$ years $(48)$

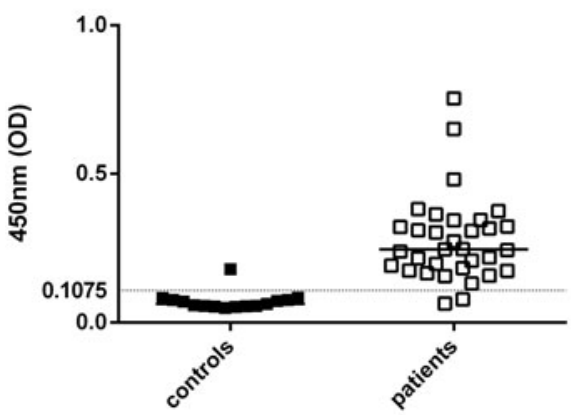

22-28 years $(60)$

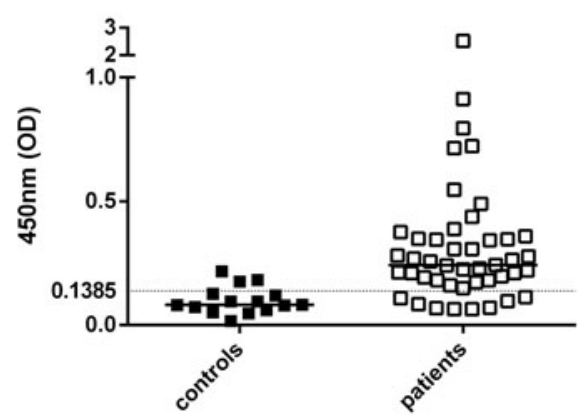

ROC Curve

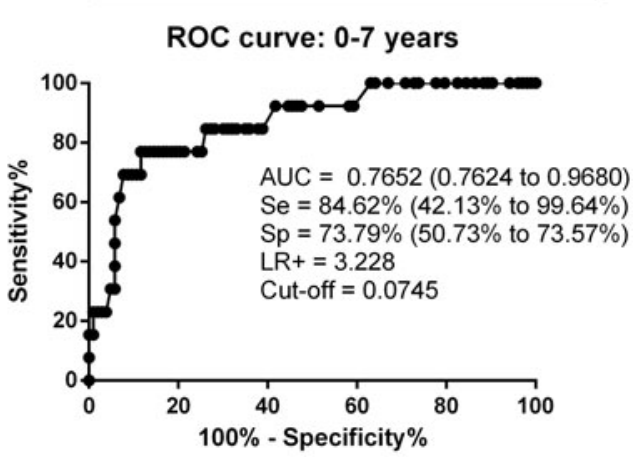

ROC curve: $\mathbf{8 - 1 4}$ years

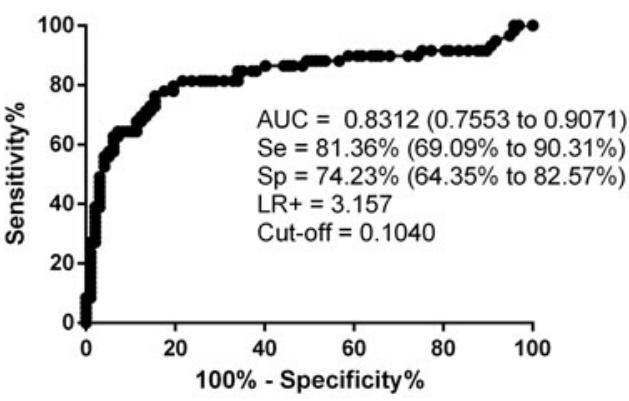

ROC curve: $15-21$ years

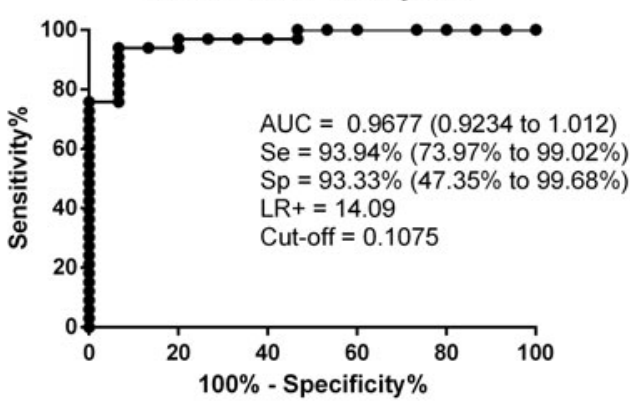

ROC curve: $\mathbf{2 2 - 2 8}$ years

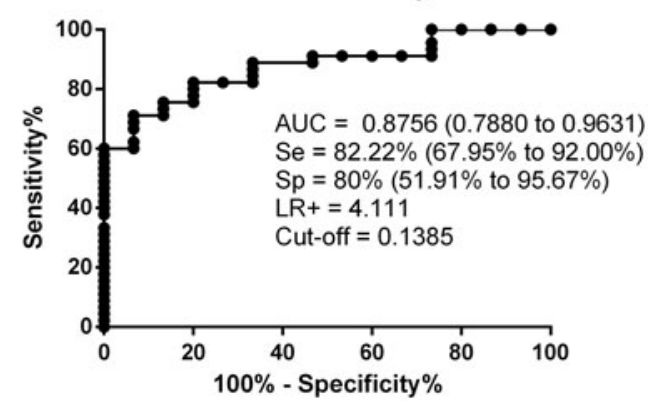

Fig. $1 b$ (cont.)

\section{DISCUSSION}

The aim of this study was to contribute to the better understanding of the relative epidemiological importance of different modes of infection with respect to horizontal transmission of $T$. gondii, namely by ingestion of tissue cysts in undercooked or uncooked meat, or by ingestion of food or water contaminated with sporulated oocysts (containing the infective sporozoite 

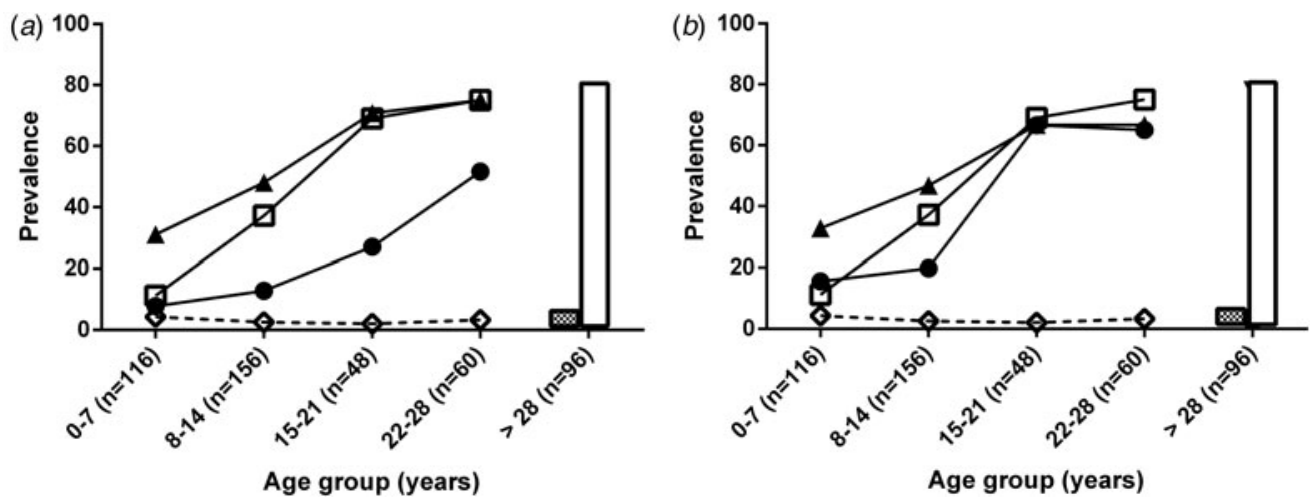

Fig. 2. T. gondii seroprevalence according to age, as determined by conventional ELISA and sporozoite-specific embryogenesis-related protein (TgERP) ELISA for all 476 individuals enrolled in this study. Data are expressed as percentages for all curves. (a) Seroprevalences according to age assessed in sera samples by $\operatorname{IgG}$ anti- $T$. gondii conventional ELISA ( $\square$ ), IgG TgERP s.D. cut-off $(\boldsymbol{\bullet})$, IgG TgERP ROC cut-off $(\boldsymbol{\Delta})$ and IgM anti-T.gondii conventional ELISA $(\diamond)$. The bars represent the seroprevalence of IgM (black bars) and IgG (white bars) in the $>28$ years age group. (b) Seroprevalence according to age assessed in saliva samples by IgG anti-T. gondii conventional ELISA ( $\square$ ), IgA TgERP S.D. cut-off $(\bullet)$, IgA TgERP ROC cut-off $(\boldsymbol{\Delta})$ and IgM anti-T.gondii conventional ELISA $(\diamond)$. The bars represent the seroprevalences of IgM (white bars with black circles) and IgG (white bars) in the $>28$ years age group. The number of individuals in each group is denoted within parentheses. The subjects included in control groups were those that tested negative for conventional ELISA against $T$. gondii $(\mathrm{IgG}$ and or $\operatorname{IgM})$ and varied according to age group: $0-7$ years $(n=$ 104 subjects), 8-14 years $(n=99), 15-21$ years $(n=15)$, and 22-28 years $(n=15)$.

forms) from cat faeces. The present findings will be discussed from an epidemiological perspective highlighting the potential value of salivary IgA against TgERP as a tool to investigate the predominant transmission route (oocysts or tissue cysts) of $T$. gondii infection in epidemiological surveys.

The disparities of prevalence rates found for each studied age group employing two different cut-off parameters shown in Table 1 provide evidence that more population-based studies employing $T$. gondii sporozoite antigens must to be conducted to improve our understanding. With the exception of the prevalence calculated for the 15-21 years age group employing salivary IgA using both s.D. and ROC cutoffs and IgG employing sera samples using the S.D. cut-off (Table 1), all other prevalences calculated using positivity against TgERP presented considerable disparities with prevalence calculated by IgG (alone or together with $\operatorname{IgM}$ ) detected by commercial kits. Nonetheless, the present study indeed confirms the importance of oocyst infection contributing to the overall prevalence of toxoplasmosis in endemic settings as shown in Table 1 and Figure 2( $a, b)$ and reported previously [30]. To the best of our knowledge this is the first report on salivary IgA against sporulated oocyst antigens.

Despite the increasing interest and the potential use of antibodies (mainly $\operatorname{IgG}$ and $\operatorname{IgA}$ ) in oral fluid (saliva), in view of its advantage over serum as a medium for antibody detection due to non-invasive sampling, the use of saliva samples for community-based epidemiological surveys, and for clinical diagnosis is in part complex and restricted to certain conditions. A comprehensive and detailed review on the physiological, immunological and technical aspects of the salivary antibodies' capacity to reproduce reliably both mucosal and systemic immunity highlights the dimension of the complexity regarding this matter [31]. Our data illustrate in part such complexity and point to the importance of the age-range parameter for saliva sampling in population-based studies on $T$. gondii infections. The age of patients was the determining factor in our study for data interpretation on the relative contribution of both $T$. gondii cysts and oocyst transmission. In fact, age has been shown to be an important variable in studies of immunoglobulins in oral fluids in general [32-34] and this phenomenon can be in part due to the slow development of the salivary IgA system [32].

In this study, because there is no serological parameter in the gold standard category for Ig recognition of T. gondii sporozoite antigens, we assumed as infected with $T$. gondii those patients presenting positive serology to commercial conventional kits that detected antibodies (IgG and or IgM against $T$. gondii) in order to analyse and compare the serology and 
salivary Ig recognition profile against $T$. gondii sporozoite antigens. Therefore, it is not possible to rule out the possibility of patients presenting as negative in conventional serology to $T$. gondii, but presenting as positive against TgERP, being infected with $T$. gondii. In fact, using the threefold s.D. criteria to establish positivity and negativity to TgERP in the present study we did not identify patients with such serological profile, namely negative to conventional serology and positive to TgERP. However, when our group used the same threefold S.D. criteria to evaluate serological IgG positivity against TgERP in a previous study, we identified patients presenting that profile [30]. We have proposed a possible explanation for that based on the vast genetic diversity of parasites in South America compared to the lower genetic diversity of parasites reported in North America and Europe [35]. Moreover, considering that commercial kits have been prepared with antigens derived from strains prevalent in North America and Europe there is in theory, a possibility of the existence of patients infected with parasites able to induce an immune response that will not recognize antigens present in commercial kits [30].

Based on threefold S.D. and ROC curve criteria that were employed to attempt to establish good cut-off values for the TgERP ELISA system in the present study it was possible to identify an age group that was shown to be suitable for studying the specific salivary antibodies against sporozoite antigens. That age group was 15-21 years, which, beyond presenting the highest values for sensitivity, specificity, and likelihood compared to all other age groups (Fig. 1), was in the prevalence curve (Fig. 2). The section of the curve where the slope is greatest is classically considered in epidemiology to be the point at which the incidence of transmission is highest. Furthermore, for that age group the best value was observed for Spearman's correlation $(0.56, P<0.0001)$ between sera $\operatorname{IgG}$ and saliva IgA against TgERP, showing that IgA from saliva against sporozoite antigens probably reflects the systemic $\operatorname{IgG}$ response against the same antigens in those aged 15-21 years. We did not evaluate salivary IgG nor serological IgA against TgERP in the present study due to scarcity of material (respectively saliva and sera) from some younger age groups. Nonetheless, it can be evaluated in further studies and will be of help to contribute to the better understanding of the mucosal, salivary glands, and systemic immune response dynamics against $T$. gondii sporozite antigens in humans. The very close prevalence rates identified using TgERP ELISAs by both cut-off criteria, namely $66 \cdot 6 \%$ by threefold S.D. criteria or $66.7 \%$ by ROC curve criteria, and $68.7 \%$ using the conventional ELISA system, reinforces the importance of the 15-21 years age group for studying the mode of transmission of $T$. gondii infection. However, it is important to note that it is likely that the age where the slope is highest in prevalence curves for toxoplasmosis is the real point of importance and studies in other areas may demonstrate that this age range can vary. The meaning of the coincidence of prevalence rates in this age group using both TgERP and tachyzoite antigens remains to be investigated. Nonetheless, it has been demonstrated that IgG against TgERP declines 8 months after primary oocyst exposure in the context of infections occurring in outbreaks and that tachyzoite immune responses detected by conventional ELISA are concomitant during this period [18]. The coincident prevalence observed using both Ig against TgERP and against tachyzoites can be explained by the possibility of a recent infection by oocysts (at least within the time-frame of 8 months). This result emphasizes the importance of studying the mode of $T$. gondii infection transmission in this age group or alternatively in the section of the age prevalence curve where the highest slope is observed.

In this study, a very low frequency of IgM-positive individuals from all age groups was observed. However, low IgM prevalence is a common factor observed for individuals living in endemic areas (including the recently infected young) for parasitic diseases [36, 37].

In conclusion, we present evidence that salivary $\operatorname{Ig} \mathrm{A}$ against sporozoite antigens is important in studying cysts $v$. oocyst-induced $T$. gondii infection in endemic settings. The major areas of importance are: $(a)$ the highest scores (sensitivity sensibility and likelihood) compared to serological IgG for all age groups studied; $(b)$ important and significant Spearman correlation value to systemic $\operatorname{IgG}(r=0.56, P<0.0001)$; and $(c)$ its identification as an $\operatorname{IgG}$ revealing $T$. gondii prevalence at the point along the age prevalence curve where the slope is the highest (for ages 14-21 years in the current study) in coincidence with systemic $\operatorname{IgG}$ against TgERP and IgG against $T$. gondii tachyzoite antigens. We propose that other endemic areas should be studied with comparable parameters as those investigated in this study with a view to clarifying if the phenomenon we describe in this study can also be observed in other endemic settings for $T$. gondii infection. Such 
replica studies would be very important for the better understanding of the relative epidemiological importance of different modes of infection with respect to horizontal transmission of $T$. gondii, which in a previous study [30], and in this study was $>50 \%$ of the total prevalence when measured by antibodies (IgG from sera or IgA from saliva) against TgERP. Equally important in such population-based studies is the potential contribution they can provide towards the recommendation of using salivary $\operatorname{IgA}$ to investigate prevalence of toxoplasmosis, with regard to its advantage over serum as a medium for antibody detection due to its non-invasive nature. Furthermore, studies of this nature are of fundamental importance to define better and more effective primary prevention strategies to minimize $T$. gondii transmission mainly in endemic settings.

\section{ACKNOWLEDGEMENTS}

We thank the educational authorities and school directors of Campos dos Goytacazes (Joilza Rangel Abreu); the public health authorities (Dr Francisco Arthur de Souza Oliveira and Charbel Curry); we are indebted to Alba L. P. Rangel Juliana Azevedo, Liliani de Souza Elias, Livia Mattos Martins, Marcela Santana Bastos Boechat, Maycon Bruno de Almeida, Rebeka da Conceição Souza, and Nubia Alencar Fernando Lopes for technical and field support. We thank ASTRAN-UENF team for field work support.

This work was supported by FAPERJ (grant nos. E-26/110·869/2009, E-26/111·305/2010, E-26/111·816/ 2013). F.P.V. was a fellow at USDA, supported by a CAPES scholarship (PDSE Process no. 709013-7).

\section{DECLARATION OF INTEREST}

None.

\section{REFERENCES}

1. Sepúlveda-Arias JC, et al. Toxoplasmosis as a travel risk. Travel Medicine and Infectious Disease 2014; 12: 592-601.

2. Dubey JP, et al. Toxoplasmosis in humans and animals in Brazil: high prevalence, high burden of disease, and epidemiology. Parasitology 2012; 139: 1375-1424.

3. Innes EA. A brief history and overview of Toxoplasma gondii. Zoonoses and Public Health 2010; 57: 1-7.

4. Desmonts G, Couvreur J. Congenital toxoplasmosis. A prospective study of 378 pregnancies. New England Journal of Medicine 1974; 290: 1110-1116.
5. Peyron F et al. Toxoplasmosis. In: Remington JS et al., eds. Infectious Diseases of the Fetus and Newborn. Philadelphia: Saunders, 2015, pp. 949-1048.

6. Gotteland C, et al. Spatial distribution of Toxoplasma gondii oocysts in soil in a rural area: influence of cats and land use. Veterinary Parasitology 2014; 205: 629-637.

7. Guo M, et al. Prevalence and risk factors for Toxoplasma gondii infection in meat animals and meat products destined for human consumption. Journal of Food Protection 2015; 78: 457-476.

8. Hide G, et al. Evidence for high levels of vertical transmission in Toxoplasma gondii. Parasitology 2009; 136: 1877-1885.

9. Hill DE, Dubey JP. Toxoplasma gondii prevalence in farm animals in the United States. International Journal for Parasitology 2013; 43: 107-113.

10. Krueger WS, et al. Drinking water source and human Toxoplasma gondii infection in the United States: a cross-sectional analysis of NHANES data. BMC Public Health 2014; 14: 711.

11. Opsteegh M, et al. Direct detection and genotyping of Toxoplasma gondii in meat samples using magnetic capture and PCR. International Journal of Food Microbiology 2010; 139: 193-201.

12. Tenter AM. Toxoplasma gondii in animals used for human consumption. Memórias do Instituto Oswaldo Cruz 2009; 104: 364-369.

13. Dubey JP. Comparative infectivity of oocysts and bradyzoites of Toxoplasma gondii for intermediate (mice) and definitive (cats) hosts. Veterinary Parasitology 2006; 140: 69-75.

14. Jiang W, et al. An agent-based model for the transmission dynamics of Toxoplasma gondii. Journal of Theoretical Biolology 2012; 293: 15-26.

15. Smith G. Food- and water-borne disease: using case control studies to estimate the force of infection that accounts for primary, sporadic cases. Epidemics 2013; 5: $77-84$.

16. Van Wormer E, et al. Molecules to modeling: Toxoplasma gondii oocysts at the human-animalenvironment interface. Comparative Immunology, Microbiology \& Infectious Diseases 2013; 36: 217-231.

17. Jones JL, Dubey JP. Waterborne toxoplasmosisrecent developments. Experimental Parasitology 2010; 124: $10-25$.

18. Hill D, et al. Identification of a sporozoite-specific antigen from Toxoplasma gondii. Journal of Parasitology 2011; 97: 328-337.

19. Santana SS, et al. CCp5A protein from Toxoplasma gondii as a serological marker of oocyst-driven infections in humans and domestic animals. Frontiers in Microbiology 2015; 6: 1305.

20. Correa D, et al. Congenital and acquired toxoplasmosis: diversity and role of antibodies in different compartments of the host. Parasite Immunology 2007; 29: 651-660.

21. Loyola AM, et al. Anti-Toxoplasma gondii immunoglobulins A and G in human saliva and serum. Journal of Oral Pathology \& Medicine 1997; 26: 187-191. 
22. Lynch MI, et al. Anti-Toxoplasma gondii secretory IgA in tears of patients with ocular toxoplasmosis: immunodiagnostic validation by ELISA. Memórias do Instituto Oswaldo Cruz 2009; 104: 818-822.

23. Chahed Bel-Ochi N, Bouratbine A, Mousli M. Enzymelinked immunosorbent assay using recombinant SAG1 antigen to detect Toxoplasma gondii-specific immunoglobulin $G$ antibodies in human sera and saliva. Clinical and Vaccine Immunology 2013; 20: 468-473.

24. Sampaio BFC, et al. Saliva as a source of antiToxoplasma gondii $\mathrm{IgG}$ for enzyme immunoassay in human samples. Clinical Microbiology and Infection 2013; 20: 72-74.

25. Oliveira AC de M, et al. Evaluation of colostrum as an alternative biological sample for the diagnosis of human congenital toxoplasmosis. BMC Infectious Diseases 2015; 15: 519.

26. Levi GC, et al. Observações complementares sôbre a presença do Toxoplasma gondii na saliva de pacientes com toxoplasmose. Revista da Sociedade Brasileira de Medicina Tropical 1968; 2: 275-278.

27. Amendoeira MR, Coutinho SG. Isolation of Toxoplasma gondii from the saliva and tonsils of a three-year-old child. Journal of Infectious Diseases 1982;

28. Bahia-Oliveira LMG, et al. Highly endemic, waterborne toxoplasmosis in north Rio de Janeiro state, Brazil. Emerging Infectious Diseases 2003; 9: 55-62.

29. Bahia-Oliveira L, et al. Toxoplasmosis-recent advance. In: Immunological and immunogenetic parameters on the diversity of ocular toxoplasmosis: evidence to support morphological criteria to classify retinallretinochoroidal scar lesions in epidemiologic surveys. Tech, Rijeka, 2012, pp. 145-172.

30. Vieira FP, et al. Waterborne toxoplasmosis investigated and analysed under hydrogeological assessment: new data and perspectives for further research. Memórias do Instituto Oswaldo Cruz 2015; 110: 929-935.

31. Brandtzaeg P. Do salivary antibodies reliably reflect both mucosal and systemic immunity? Annals of the New York Academy of Sciences; 2007; 1098: 288-311.

32. Brandtzaeg P. Synthesis and secretion of human salivary immunoglobulins. In: Garrett JR, Ekström J, Anderson LC, eds. Glandular Mechanisms of Salivary Secretion. Basel: Karger, 1998, pp. 167-199.

33. Sonesson M, et al. Salivary IgA in minor-gland saliva of children, adolescents, and young adults. European Journal of Oral Sciences 2011; 119: 15-20.

34. Jafarzadeh A, et al. Salivary $\operatorname{IgA}$ and $\operatorname{IgE}$ levels in healthy subjects: relation to age and gender. Brazilian Oral Research 2010; 24: 21-27.

35. Shwab EK, et al. Geographical patterns of Toxoplasma gondii genetic diversity revealed by multilocus PCRRFLP genotyping. Parasitology 2014; 141: 453-461.

36. Eloi-Santos ASM, et al. Idiotypic sensitization in utero of children born to mothers with schistosomiasis or Chagas' disease. Journal of Clinical Investigation 1989; 84: 1028-1031.

37. King CL, et al. Acquired immune responses to Plasmodium falciparum merozoite surface protein-1 in the human fetus. Journal of Immunology 2002; 168: 356-64. 